\title{
EXPLICIT MIN-MAX MODEL PREDICTIVE CONTROL OF CONSTRAINED NONLINEAR SYSTEMS WITH MODEL UNCERTAINTY
}

\author{
Alexandra Grancharova ${ }^{1,2,3}$ and Tor A. Johansen ${ }^{4}$ \\ Department of Engineering Cybernetics, Norwegian University of Science and \\ Technology, N-7491 Trondheim, Norway
}

\begin{abstract}
This paper presents an approximate multi-parametric nonlinear programming approach to explicit solution of constrained nonlinear model predictive control (MPC) problems in the presence of model uncertainty. The case of time-invariant parameter uncertainty is considered. The explicit MPC controller is based on an orthogonal search tree structure of the state space partition and is designed by solving a min-max optimization problem. It is robust in the sense that all constraints are satisfied for all possible values of the uncertain parameters. The approach is applied to design an explicit min-max MPC controller for a continuous stirred tank reactor, where the heat transfer coefficient is an uncertain parameter. Copyright (C) 2005 IFAC
\end{abstract}

Keywords: predictive control, nonlinear systems, constraints, uncertainty, piecewise linear controllers.

\section{INTRODUCTION}

Model predictive control (MPC) is an efficient methodology to solve complex constrained multivariable control problems in the absence, as well as in the presence of uncertainties (Mayne et al., 2000). The requirement to perform on-line optimization, however, restricts the applicability of MPC to relatively slow processes. Recently, several methods for explicit solution of MPC problems have been developed. The main motivation behind explicit MPC is that an explicit state feedback law avoids the need for real-time optimization, and is therefore potentially useful for applications with fast sampling where MPC has not traditionally been used.

\footnotetext{
1 This work was sponsored by the Research Council of Norway (Project "Explicit solution of model predictive control problems in the presence of model uncertainty", 160249/V30/ntm)

${ }^{2}$ Present address: Institute of Control and System Research, Bulgarian Academy of Sciences, Acad. G. Bonchev str., Bl.2, P.O.Box 79, Sofia 1113, Bulgaria

3 alexandra@icsr.bas.bg

${ }^{4}$ Tor.Arne.Johansen@itk.ntnu.no
}

In (Bemporad et al., 2002; Seron et al., 2000; Tøndel et al., 2003) multi-parametric quadratic programming (mp-QP) algorithms for explicit solution of constrained linear MPC problems have been proposed. In (Johansen and Grancharova, 2003; Grancharova and Johansen, 2002), algorithms that determine an approximate explicit piecewise linear (PWL) state feedback solution by imposing an orthogonal search tree structure on the partition, have been developed. They may lead to even more efficient real-time computations. Some of the parametric programming approaches have been further extended to ensure robustness of the explicit MPC controllers against bounded additive or polytopic uncertainties (Bemporad et al., 2003; Kerrigan and Maciejowski, 2003; Sakizlis et al., 2004; Grancharova and Johansen, 2003; Muñoz de la Peña et al., 2004).

The methods mentioned above are applied to linear systems. Recently, approximate approaches to explicit solution of constrained nonlinear MPC problems have been developed in (Johansen, 2002; Johansen, 2004). They are based on the multi-parametric nonlinear programming (mp-NLP) technique described in (Fiacco, 1983). However, these approaches assume that the process model is known exactly. Since 
models are only an approximation of the real process, it is important for MPC to be robust to model uncertainty.

This paper suggests an approximate mp-NLP approach to explicit solution of constrained nonlinear MPC problems in the presence of model uncertainty. It is based on an orthogonal search tree structure of the state space partition and thus represents an extension of the approach in (Johansen, 2004). The explicit MPC controller is designed by solving a minmax optimization problem, i.e. by minimizing the worst-case with respect to the uncertain parameters cost function value. The controller is robust in the sense that all constraints are satisfied for all possible values of the uncertain parameters.

\section{FORMULATION OF MIN-MAX MODEL PREDICTIVE CONTROL PROBLEM}

Consider the discrete-time nonlinear system:

$$
\begin{aligned}
& x(t+1)=f(x(t), u(t), \theta) \\
& y(t)=C x(t)
\end{aligned}
$$

where $x(t) \in \Re^{n}, u(t) \in \mathfrak{R}^{m}$, and $y(t) \in \mathfrak{R}^{p}$ are the state, input and output variable, $\theta$ is the vector of time-invariant uncertain parameters that is assumed to belong to a bounded polyhedral set $\theta \in \Theta^{A} \subset \mathfrak{R}^{s}$. It is also assumed that the function $f$ is sufficiently smooth. It is supposed that a full measurement of the state $x(t)$ is available at the current time $t$. We consider the following open-loop robust MPC problem: For the current $x(t)$, MPC minimizes the worst-case cost function through the following optimization:

$$
V_{\max }^{*}(x(t))=\min _{U} \max _{\theta \in \Theta^{A}} J(U, x(t), \theta)
$$

subject to $x_{t \mid t}=x(t)$ and:

$$
\begin{aligned}
& y_{\min } \leq y_{t+k \mid t} \leq y_{\max }, \forall \theta \in \Theta^{A}, k=1, \ldots, N \\
& u_{\min } \leq u_{t+k} \leq u_{\max }, k=0,1, \ldots, N-1 \\
& x_{t+N \mid t}^{T} x_{t+N \mid t} \leq \delta, \forall \theta \in \Theta^{A} \\
& x_{t+k+1 \mid t}=f\left(x_{t+k \mid t}, u_{t+k}, \theta\right), \theta \in \Theta^{A}, k \geq 0 \\
& y_{t+k \mid t}=C x_{t+k \mid t}, k \geq 0
\end{aligned}
$$

with $U=\left\{u_{t}, u_{t+1}, \ldots, u_{t+N-1}\right\}$ and the cost function given by:

$$
\begin{gathered}
J(U, x(t), \theta)=\sum_{k=0}^{N-1}\left[x_{t+k \mid t}^{T} Q x_{t+k \mid t}+u_{t+k}^{T} R u_{t+k}\right] \\
+x_{t+N \mid t}^{T} P x_{t+N \mid t}
\end{gathered}
$$

Here, $N$ is a finite horizon. In (3), the existence of minimum and maximum are implicitly assumed. From a stability point of view it is desirable to choose $\delta$ in (6) as small as possible (Mayne et al., 2000). However, due to the fact that $x_{t+N \mid t}$ depends on the unknown $\theta$, the feasibility of (3)-(9) will rely on $\delta$ being sufficiently large. A part of the MPC design will be to address this tradeoff. If the system is asymptotically stable (or pre-stabilized), $N$ is large, and possibly an integral action is introduced to account for the steady-state effect of the uncertainty, then it is more likely that the choice of a small $\delta$ will be possible.

The following assumptions are made:

A1. $P, Q, R \succ 0$.

A2. $y_{\text {min }}<0<y_{\text {max }}$.

A3. $\theta$ is time-invariant uncertainty that belongs to a bounded polyhedral set, i.e. $\theta=$ const $\in \Theta^{A}$. The polyhedral set $\Theta^{A}$ is defined by $\Theta^{A}=\left\{\theta \in \mathfrak{R}^{s} \mid \theta^{L} \leq \theta \leq \theta^{U}\right\}$, where $\theta^{L}$ and $\theta^{U}$ represent given lower and upper bounds on $\theta$.

A4. For each $\theta \in \Theta^{A}$ there exists $u_{s t} \in \mathfrak{R}^{m}$ satisfying $u_{\text {min }} \leq u_{s t} \leq u_{\text {max }}$, and such that $f\left(0, u_{s t}, \theta\right)=0$.

Assumption A4 means that the point $x=0, u=u_{s t}$ is a steady state point for system (1). It also implies that the steady state value of the control input will be different for the different values of the uncertain parameters.

The worst-case value of cost function (9) with respect to the uncertain parameters is denoted by:

$$
V_{\max }(U, x(t))=\max _{\theta \in \Theta^{A}} J(U, x(t), \theta)
$$

An optimal solution to the min-max MPC problem (3)-(9) is denoted $U^{*}=\left\{u_{t}^{*}, u_{t+1}^{*}, \ldots, u_{t+N-1}^{*}\right\}$ and the control input is chosen according to the receding horizon policy $u(t)=u_{t}^{*}$. The optimization problem can be formulated in a compact form as follows:

$$
V_{\text {max }}^{*}(x(t))=\min _{U} \max _{\theta \in \Theta^{A}} J(U, x(t), \theta)
$$

subject to:

$$
G(U, x(t), \theta) \leq 0, \forall \theta \in \Theta^{A}
$$

This min-max MPC problem defines an mp-NLP, since it is NLP in $U$ parameterized by $x$. Define the set of $N$-step robustly feasible initial states as follows:

$$
\begin{gathered}
X_{f}=\left\{x \in \mathfrak{R}^{n} \mid G(U, x, \theta) \leq 0, \forall \theta \in \Theta^{A}\right. \\
\text { for some } \left.U \in \mathfrak{R}^{N m}\right\}
\end{gathered}
$$

If assumption A4 is satisfied and $\delta$ is chosen such that the problem (3)-(9) is feasible, then $X_{f}$ is a nonempty set. Then, due to assumption A2, the origin is an interior point in $X_{f}$.

\section{APPROXIMATE MP-NLP APPROACH TO EXPLICIT MIN-MAX MODEL PREDICTIVE CONTROL}

\subsection{Feasibility in the presence of model uncertainty.}

The numerical computations involved in constructing the approximate explicit state feedback are simplified under the following convexity assumption: 
A5. $G(U, x, \theta)$ is jointly convex for all $(U, x, \theta) \in U^{A} \times X \times \Theta^{A}$, where $U^{A}=\left[u_{\text {min }}, u_{\text {max }}\right]^{N}$ is the set of admissible inputs.

We exploit the result in (Grossmann et al., 1983), where it has been shown that if the constraint function $G(U, x, \theta)$ is jointly convex in $U$ and $\theta$, and there is

$U$ that is feasible at the vertices of $\Theta^{A}$, then $U$ is feasible for all $\theta \in \Theta^{A}$. This is formulated in the following lemma:

\section{Lemma 1:}

Suppose $A 3$ and $A 5$ hold and denote the vertices of the polyhedron $\Theta^{A} \subset \Re^{s}$ with $\left\{\theta_{1}, \theta_{2}, \ldots, \theta_{L}\right\}$. Denote also $\tilde{G}^{i}(U, x)=G\left(U, x, \theta_{i}\right)$. If there exist $U$ that satisfies the following constraints:

$$
\widetilde{G}^{i}(U, x) \leq 0, i \in\{1,2, \ldots, L\}
$$

then $U$ satisfies constraint (12).

Thus, we can replace the infinite number of constraints (12) with the following finite amount of jointly convex constraints which are function only of $U$ and $x$ :

$$
\widetilde{G}(U, x) \leq 0, \tilde{G}(U, x)=\left\{\tilde{G}^{i}(U, x), i=1,2, \ldots, L\right\}
$$

Then, the above problem can be formulated as:

$$
\begin{aligned}
& V_{\max }^{*}(x)=\min _{U} V_{\max }(U, x) \\
& \text { subject to } \widetilde{G}(U, x) \leq 0
\end{aligned}
$$

where $V_{\max }(U, x)$ is defined by (10). The following assumption is made:

A6. $V_{\max }(U, x)$ is jointly convex for all $(U, x) \in U^{A} \times X$.

The optimal solution to problem (16) will be denoted $U^{*}(x)$. We restrict our attention to a hyper-rectangle $X \subset R^{n}$ where we seek to approximate the optimal solution $U^{*}(x)$ to the mp-NLP (16). In order to minimize the real-time computational complexity we require that the state space partition is orthogonal and can be represented as a $k-d$ tree (Bentley, 1975; Grancharova and Johansen, 2002). The main idea of the approximate mp-NLP approach is to construct a robustly feasible piecewise linear (PWL) approximation $\hat{U}(x)$ to $U^{*}(x)$ on $X$, where the constituent affine functions are defined on hyperrectangles covering $X$. For this purpose, the solution of the problem (16) is computed at the $2^{n}$ vertices of a considered hyper-rectangle $X_{0}$ by solving up to $2^{n}$ NLPs. Based on these solutions, a robustly feasible local linear approximation $\hat{U}_{0}(x)=K_{0} x+g_{0}$ to the optimal solution $U^{*}(x)$, valid in the whole hyperrectangle $X_{0}$, is determined. Let $\hat{V}_{\max , 0}(x)$ denotes the worst-case value of the cost function (9) obtained with this approximation, i.e.:

$$
\hat{V}_{\max , 0}(x)=V_{\max }\left(K_{0} x+g_{0}, x\right)
$$

Then, the robustly feasible local linear feedback law $\hat{U}_{0}(x)=K_{0} x+g_{0}$ is computed by applying the following lemma:

\section{Lemma 2:}

Suppose A1-A6 hold, and consider any bounded polyhedron $X_{0} \subseteq X_{f}$ with vertices $\left\{v_{1}, v_{2}, \ldots, v_{M}\right\}$. If

$K_{0}$ and $g_{0}$ solve the convex NLP:

$$
\begin{aligned}
\min _{K_{0}, g_{0}} \sum_{i=1}^{M} & \left(\hat{V}_{\max , 0}\left(v_{i}\right)-V_{\max }^{*}\left(v_{i}\right)\right. \\
& \left.+\beta\left\|K_{0} v_{i}+g_{0}-U^{*}\left(v_{i}\right)\right\|_{2}^{2}\right)
\end{aligned}
$$

subject to:

$$
\tilde{G}\left(K_{0} v_{i}+g_{0}, v_{i}\right) \leq 0, i \in\{1,2, \ldots, M\},
$$

then $\hat{U}_{0}(x)=K_{0} x+g_{0}$ is feasible for the $m p-N L P$ (16) for all $x \in X_{0}$.

Proof:

It follows from Lemma 2 in (Johansen, 2004). $\square$

\subsection{Error bounds}

It can be shown that the optimal cost function has the following properties (Mangasarian and Rosen, 1964; Johansen, 2004):

\section{Theorem 1:}

Suppose A1-A6 hold. Then $X_{f}$ is a closed convex set, and $V_{\max }^{*}: X_{f} \rightarrow \Re$ is convex and continuous.

The accuracy of approximation will be measured by the difference between the optimal and sub-optimal cost functions. Since the optimal cost function $V_{\text {max }}^{*}(x)$ can not be assumed known, convexity may be exploited to compute simple bounds to be used for constructing the approximate solution, similar to (Fiacco, 1983). Consider the vertices $\left\{v_{1}, v_{2}, \ldots, v_{M}\right\}$ of any bounded polyhedron $X_{0} \subseteq X_{f}$. Define the affine function $\bar{V}(x)=\bar{V}_{0} x+\bar{l}_{0}$ as the solution to the following linear program (LP):

$$
\min _{\bar{V}_{0}, \bar{l}_{0}}\left(\bar{V}_{0} v+\bar{l}_{0}\right)
$$

subject to:

$$
\bar{V}_{0} v_{i}+\bar{l}_{0} \geq V_{\text {max }}^{*}\left(v_{i}\right) \text { for all } i \in\{1,2, \ldots, M\}
$$

Likewise, define the convex PWL function:

$$
\underline{V}(x)=\max _{i \in\{1,2, \ldots, M\}}\left(V_{\max }^{*}\left(v_{i}\right)+\nabla^{T} V_{\max }^{*}\left(v_{i}\right)\left(x-v_{i}\right)\right)
$$

If $V_{\max }^{*}(x)$ is not differentiable at $v_{i}$, then $\nabla V_{\max }^{*}\left(v_{i}\right)$ is taken as any sub-gradient of $V_{\max }^{*}(x)$ at $v_{i}$ (Rockafellar, 1970). Then, it can be shown that $\bar{V}(x)$ and $\underline{V}(x)$ have the following properties (Fiacco, 1983; Johansen, 2004):

\section{Theorem 2:}

Suppose A1-A6 hold, and consider any bounded polyhedron $X_{0} \subseteq X_{f}$. Then $\underline{V}(x) \leq V_{\max }^{*}(x) \leq \bar{V}(x)$ for all $x \in X_{0}$. 
Since $\hat{U}_{0}(x)$ defined in Lemma 2 is feasible in $X_{0}$, it follows that the sub-optimal cost $\hat{V}_{\max }(x)=V_{\max }\left(\hat{U}_{0}(x), x\right)$ is an upper bound on $V_{\max }^{*}(x)$ in $X_{0}$, such that for all $x \in X_{0}$ we have:

$$
0 \leq \hat{V}_{\max }(x)-V_{\max }^{*}(x) \leq \varepsilon_{0}
$$

where:

$$
\varepsilon_{0}=-\min _{x \in X_{0}}\left(-\hat{V}_{\max }(x)+\underline{V}(x)\right)
$$

Computing $\varepsilon_{0}$ requires the solution of the NLP (24). If $\underline{V}(x)$ is chosen as PWL (cf. (22)), this NLP is concave since $\hat{V}_{\max }(x)$ is convex. Hence, the optimization can be done efficiently since $X_{0}$ is a polyhedron and it suffices to compare the solution at its vertices due to concavity.

\subsection{Approximate $\mathrm{mp}$-NLP algorithm for explicit min- max model predictive control.}

Assume the tolerance $\bar{\varepsilon}>0$ of the cost function approximation error is given. The following algorithm is proposed to design explicit min-max MPC controller for constrained nonlinear systems with model uncertainty:

Algorithm 1 (approximate mp-NLP for explicit min-max MPC)

Step 1. Transform the original mp-NLP problem with model uncertainty (11)-(12) into the mp-NLP problem (16), by applying Lemma 1.

Step 2. Initialize the partition to the whole hyperrectangle, i.e. $P=\{X\}$. Mark the hyper-rectangle $X$ as unexplored.

Step 3. Select any unexplored hyper-rectangle $X_{0} \in P$. If no such hyper-rectangle exists, the algorithm terminates successfully.

Step 4. Compute the solution to the NLP (16) for $x$ fixed to each of the $2^{n}$ vertices of the hyper-rectangle $X_{0}$. If all NLPs have a feasible solution, go to step 6 . Otherwise, go to step 5.

Step 5. Compute the size of $X_{0}$ using some metric. If it is smaller than some given tolerance, mark $X_{0}$ infeasible and explored and go to step 3. Otherwise, go to step 8.

Step 6. Compute an affine state feedback $\hat{U}_{0}(x)$ using Lemma 2, as an approximation to be used in $X_{0}$. If no feasible solution was found, go to step 8 .

Step 7. Compute the error bound $\varepsilon_{0}$ in $X_{0}$. If $\varepsilon_{0} \leq \bar{\varepsilon}$, mark $X_{0}$ as explored and feasible and go to step 3 .

Step 8. Split the hyper-rectangle $X_{0}$ into two hyperrectangles $X_{1}$ and $X_{2}$ by applying the heuristic splitting rule from (Grancharova and Johansen, 2002). Mark them unexplored, remove $X_{0}$ from $P$, add $X_{1}$ and $X_{2}$ to $P$, and go to step 3 .
If convexity does not hold (assumptions A5 and A6), then global optimization (Horst and Tuy, 1995) should be applied in several steps of the algorithm. This can be done in a way similar to that in (Johansen, 2004):

- The NLP (16) should be solved using global optimization in step 4.

- The NLP (18)-(19) should be reformulated and solved using global optimization in step 6. It is not sufficient to impose the constraints at the vertices of the polyhedron $X_{0}$ if $\widetilde{G}$ is not convex. One approach to resolve this problem is to include some interior points in addition to the set of vertices $\left\{v_{1}, v_{2}, \ldots, v_{M}\right\}$ when used in (18)-(19).

- The computation of the error bound $\varepsilon_{0}$ in step 7 assumes the knowledge of a lower bound $\underline{V}$ (cf. (22)) on the optimal cost function. In case of nonconvexity, additional (interior) points may be used in order to obtain a sufficiently accurate estimate of the bound $\underline{V}$.

In contrast to the conventional MPC based on realtime optimization, the explicit MPC makes the rigorous verification and validation of the controller performance much easier (Johansen, 2004). Hence, problems due to lack of convexity and numerical difficulties can be addressed during the design and implementation. Notice that the off-line computational complexity and real-time computer memory requirements may grow very quickly with the number of states.

\section{SIMULATION EXAMPLE}

The proposed approach is applied to design an explicit min-max MPC controller for a continuous stirred tank reactor (CSTR) in which a first-order irreversible reaction $\mathrm{A} \rightarrow \mathrm{B}$ takes place (Figure 1 ). The mathematical model of CSTR and values of the parameters are taken from (Hicks and Ray, 1971).

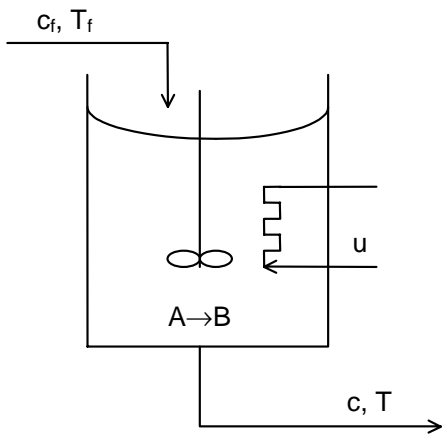

Fig. 1. Continuous stirred tank reactor.

The mass and heat balance of CSTR expressed through dimensionless concentration $\tilde{c}$ and temperature $\tilde{T}$ are:

$$
\frac{d \tilde{c}}{d t}=\frac{(1-\tilde{c})}{q}-k_{0} e^{-\frac{E}{T}} \tilde{c}
$$




$$
\frac{d \tilde{T}}{d t}=\frac{\left(\tilde{T}_{f}-\tilde{T}\right)}{q}+k_{0} e^{-\frac{E}{\tilde{T}} \tilde{c}}-\alpha u\left(\tilde{T}-\tilde{T}_{c}\right)
$$

where the dimensionless quantities $\tilde{c}, \tilde{T}, \widetilde{T}_{c}$ and $\tilde{T}_{f}$ are defined as follows:

$$
\tilde{c}=\frac{c}{c_{f}}, \tilde{T}=\frac{T}{J c_{f}}, \tilde{T}_{c}=\frac{T_{c}}{J c_{f}}, \tilde{T}_{f}=\frac{T_{f}}{J c_{f}}
$$

The coolant flowrate $u$ is the control variable. The values of the parameters are taken from (Hicks and Ray, 1971) and are $q=10, c_{f}=1, T_{c}=290$, $T_{f}=300, J=100, E=25.2, k_{0}=300$. The heat transfer coefficient $\alpha$ is an uncertain parameter that belongs to the interval:

$$
1.9 \cdot 10^{-4} \leq \alpha \leq 2.5 \cdot 10^{-4}
$$

The coolant flowrate is constrained to be:

$$
0 \leq u \leq 600
$$

We consider the set point $\tilde{c}^{*}=0.41, \tilde{T}^{*}=3.3$. Then, the model of the reactor can be written in the form:

$$
\begin{gathered}
\frac{d x_{1}}{d t}=\frac{\left(1-\tilde{c}^{*}-x_{1}\right)}{q}-k_{0} e^{-\frac{E}{\left(\tilde{T}^{*}+x_{2}\right)}}\left(\tilde{c}^{*}+x_{1}\right) \\
\frac{d x_{2}}{d t}=\frac{\left(\tilde{T}_{f}-\tilde{T}^{*}-x_{2}\right)}{q}+k_{0} e^{-\frac{E}{\left(\tilde{T}^{*}+x_{2}\right)}}\left(\tilde{c}^{*}+x_{1}\right) \\
\quad-\alpha u\left(\tilde{T}^{*}+x_{2}-\tilde{T}_{c}\right)
\end{gathered}
$$

where $x_{1}$ and $x_{2}$ denote the deviations of the concentration and temperature from the set point values $\left(x_{1}=\tilde{c}-\tilde{c}^{*}, x_{2}=\tilde{T}-\tilde{T}^{*}\right)$. We discretize the model (30), (31) using a sampling time $T_{s}=1$.

The approximate mp-NLP approach described in section 3 is applied to design an explicit min-max MPC controller for this reactor. The MPC minimizes the worst-case (maximal) value with respect to the uncertain parameter $\theta=\alpha$ of the cost function (9) subject to the system equations (30), (31) and the input constraint (29). In (9), the cost matrices are:

$$
Q=P=\operatorname{diag}\{100,300\}, R=1 \cdot 10^{-6}
$$

The horizon is $N=30$. In (6), it is chosen $\delta=0.002$. The state space to be partitioned is defined by $X=[-0.4,0.6] \times[-0.4,0.5]$. The state space partition of the min-max MPC controller is shown in Figure 2. It has 94 regions and 10 levels of search. With one scalar comparison required at each level of the $k-d$ tree, 10 arithmetic operations are required in the worst case to determine which region the state belongs to. Totally, 14 arithmetic operations are needed in realtime to compute the control input (10 comparisons, 2 multiplications and 2 additions).

The performance of the closed-loop system was simulated for initial condition $x(0)=\left[\begin{array}{ll}0.58 & 0.3\end{array}\right]^{T}$ and for three values of the uncertain parameter $\left(\alpha=1.9 \cdot 10^{-4}, \alpha=2.2 \cdot 10^{-4}, \alpha=2.5 \cdot 10^{-4}\right)$. The resulting closed-loop response is depicted in the state space (Figure 2), as well as trajectories in time
(Figure 3 to Figure 6). It can be seen that the explicit min-max MPC controller brings the reactor to the desired set point despite of the model uncertainty, and the constraints imposed on the system are satisfied. In order to avoid a possible offset, the dual-mode control strategy of (Michalska and Mayne, 1993) was applied and a locally stabilizing control law was used in a neighborhood of the origin.

The non-convexity in this example was handled by considering also internal points of the hyperrectangles in addition to vertices, as suggested in section 3.3. Also, the optimal solution to the NLP problem was determined by comparing the solutions obtained with different initial guesses for the optimization vector.

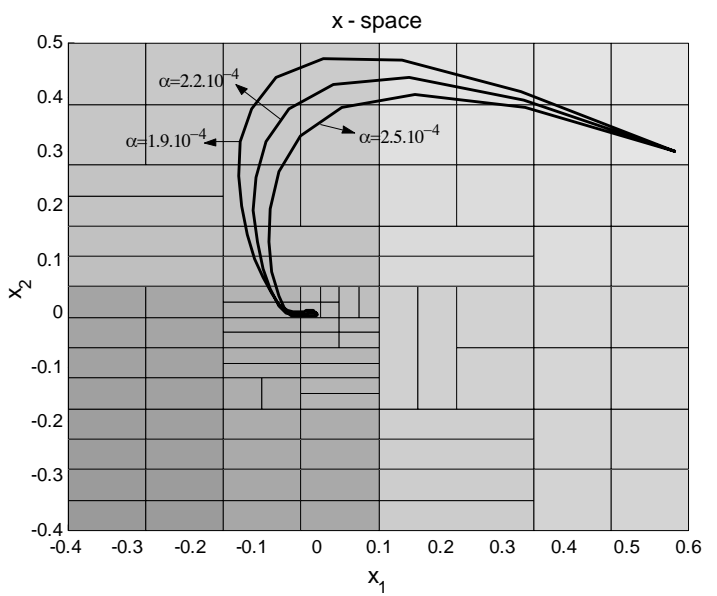

Fig. 2. State space partition of the explicit min-max MPC and the state trajectories corresponding to $\alpha=1.9 \cdot 10^{-4}, \alpha=2.2 \cdot 10^{-4}, \alpha=2.5 \cdot 10^{-4}$.

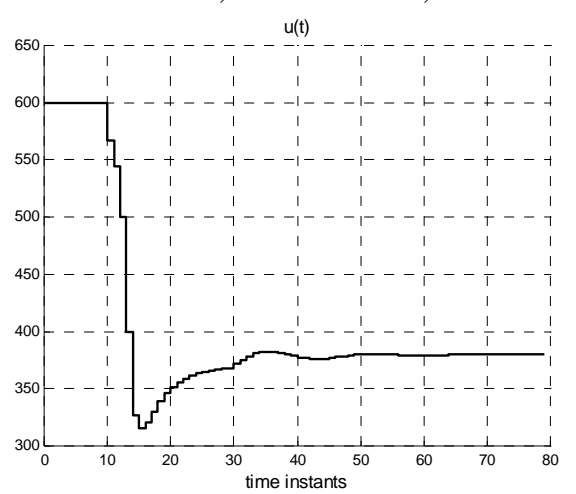

Fig. 3. Control input corresponding to $\alpha=1.9 \cdot 10^{-4}$.

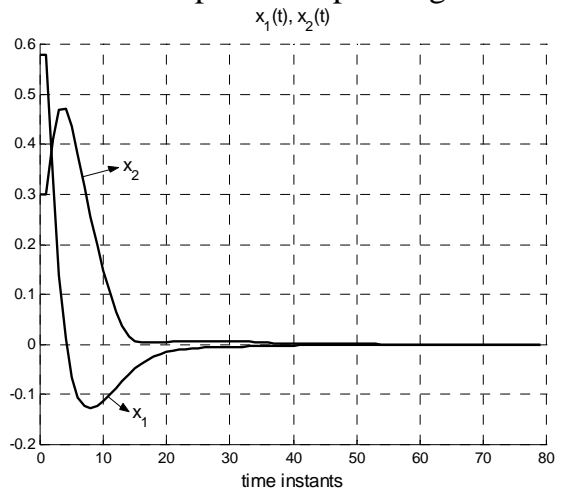

Fig. 4. State trajectory corresponding to $\alpha=1.9 \cdot 10^{-4}$. 


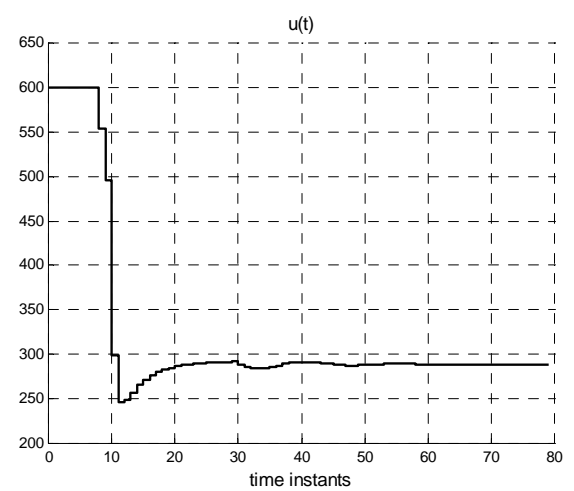

Fig. 5. Control input corresponding to $\alpha=2.5 \cdot 10^{-4}$.

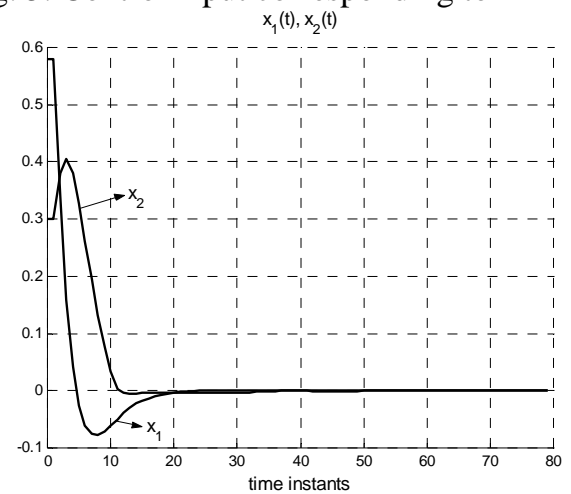

Fig. 6. State trajectory corresponding to $\alpha=2.5 \cdot 10^{-4}$.

\section{CONCLUSIONS}

An approximate mp-NLP approach to explicit solution of constrained nonlinear MPC problems in the presence of model uncertainty is suggested. The explicit MPC controller is based on an orthogonal search tree structure of the state space partition and is designed by solving a min-max optimization problem. The approach is applied to design an explicit min-max MPC controller for a continuous stirred tank reactor, where the heat transfer coefficient is an uncertain parameter.

\section{REFERENCES}

Bemporad, A., F. Borrelli and M. Morari (2003). Min-max control of constrained uncertain discrete-time linear systems. IEEE Transactions on Automatic Control, 48, 1600-1606.

Bemporad, A., M. Morari, V. Dua, and E. N. Pistikopoulos (2002). The explicit linear quadratic regulator for constrained systems. Automatica, $\mathbf{3 8 \text { , }}$ 3-20.

Bentley, J. L. (1975). Multidimensional binary search trees used for associative searching. Communications of the ACM, 18, 509-517.

Fiacco, A. V. (1983). Introduction to sensitivity and stability analysis in non-linear programming. Academic press, New York.

Grancharova, A. and T. A. Johansen (2002). Approximate explicit model predictive control incorporating heuristics. In: Proceedings of IEEE International Symposium on Computer Aided Control System Design, Glasgow, pp.92-97.
Grancharova, A. and T. A. Johansen (2003). Design of robust explicit model predictive controller via orthogonal search tree partitioning. In: Proceedings of the European Control Conference, Cambridge, U.K.

Grossmann, I. E., K. P. Halemane and R. E. Swaney (1983). Optimization strategies for flexible chemical processes. Computers and Chemical Engineering, 7, 439-462.

Hicks, G. and W. Ray (1971). Approximation methods for optimal control synthesis. The Canadian Journal of Chemical Engineering, 49, 522-528.

Horst, R. and H. Tuy (1995). Global optimization. Berlin, Springer.

Johansen, T. A. and A. Grancharova (2003). Approximate explicit constrained linear model predictive control via orthogonal search tree. IEEE Transactions on Automatic Control, 48, 810-815.

Johansen, T. A. (2002). On multi-parametric nonlinear programming and explicit nonlinear model predictive control. In: Proceedings of IEEE Conference on Decision and Control, Las Vegas, NV, vol.3, pp.2768-2773.

Johansen, T. A. (2004). Approximate explicit receding horizon control of constrained nonlinear systems. Automatica, 40, 293-300.

Kerrigan, E. C. and J. M. Maciejowski (2003). On robust optimization and the optimal control of constrained linear systems with bounded state disturbances. In: Proceedings of the European Control Conference, Cambridge, U.K.

Mangasarian, O. L. and J. B. Rosen (1964). Inequalities for stochastic nonlinear programming problems. Operations Research, 12, 143-154.

Mayne, D. Q., J. B. Rawlings, C. V. Rao and P. O. M. Scokaert (2000). Constrained model predictive control: Stability and optimality. Automatica, 36, 789-814.

Michalska, H. and D. Q. Mayne (1993). Robust receding horizon control of constrained nonlinear systems. IEEE Transactions on Automatic Control, 38, 1623-1633.

Muñoz de la Peña, D., A. Bemporad and C. Filippi (2004). Robust explicit MPC based on approximate multi-parametric convex programming. In: Proceedings of IEEE Conference on Decision and Control, Bahamas.

Rockafellar, R. T. (1970). Convex analysis, Princeton University Press, New Jersey.

Sakizlis, V., N. M. P. Kakalis, V. Dua, J. D. Perkins and E. N. Pistikopoulos (2004). Design of robust model-based controllers via parametric programming. Automatica, 40, 189-201.

Seron, M., J. A. De Dona and G. C. Goodwin (2000). Global analytical model predictive control with input constraints. In: Proceedings of IEEE Conference on Decision and Control, Sydney.

Tøndel, P., T. A. Johansen and A. Bemporad (2003). An algorithm for multi-parametric quadratic programming and explicit MPC solutions. Automatica, 39, 489-497. 